\title{
XLVIII. On the imprisonment of radiation by total reflexion
}

\section{R.W. Wood}

To cite this article: R.W. Wood (1913) XLVIII. On the imprisonment of radiation by total reflexion, Philosophical Magazine Series 6, 25:148, 449-452, DOI: 10.1080/14786440408634184

To link to this article: http://dx.doi.org/10.1080/14786440408634184

曲 Published online: 08 Jun 2010.

Submit your article to this journal ¿

Џll Article views: 3

Q View related articles $\sqsubset$ 
Prof. Michelson's gratings, in their 75-foot spectrograph ('Tower telescope), which is superior to those which I made at E. Hampton. Prof. Michelson's grating shows even more than full theoretical resolving power, and is superior to the one with which my work was done.

XLVIII. On the Imprisonment of Radiation by Total Reflexion. By R. W. Wood, Professor of Experimental Physics, Johns Hopkins University, and Adams Researdh Fellow of Columbia University*.

MY attention was recently drawn to a somewhat surprising 1 circumstance in connexion with fluorescence by Dr. Cooper Hewitt. As is well known, he has now perfected his rhodamine fluorescent reflectors for the mereury are to such a point that the red fluorescent radiation, plus the direct light of the arc, makes a fairly good imitation of daylight. He found that if the celluloid film stained with rhodamine was backed by white paper the fluorescence was many times brighter than when a backing of silver paper was used. I verified the matter by depositing silver chemically over a small circular area in the centre of a white porcelain plate, and flowing the whole with the rhodamine solution, or with water containing a little fluorescein. Notwithstanding the very high reflecting power of the polished silver fim, the intensity of the flcorescence over the circular area was so feeble that it appeared almost black in contrast with the brilliant fluorescence displayed over the surface of the porcelain. The plate was illuminated with the mercury are in the case of the rhodamine, or with that of the carbon arc, passed through dense cobalt glass in the case of the fluorescein, the experiment being well suited for lecture purposes. If we strip off the coloured celluloid film from the porcelain plater we find its fluorescence becomes even less brilliant than that. of the portion backed by silver. The action of the whits porcelain surface in raising the intensity of the fluorescence of the film covering it appeared a little puzzling at first. It was noticed, however, that the films which had been stripped from the surface glowed brilliantly along the edges, and this circumstance gave me the clue to the action of the matt reflecting surface. The greater luminosity of the elges was evidently due to the circumstance that more radiation was able to escape here than through an equal area of the surface of the film. The action of the matt surface will bc

* Communicated by the Author.

Phil. Mag. S. 6. Vol. 25. No. 148. April 1913. 
clear from reference to fig. 1 , in which $A B$ represents the upper, and CD the lower surface of a celluloid film stained

Fig. 1.

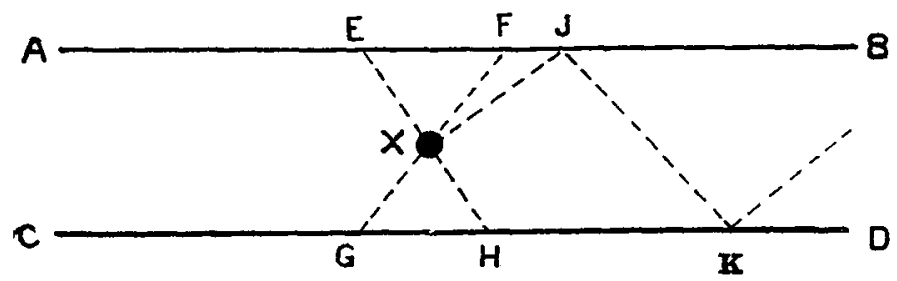

with rhodamine. Let $\mathrm{X}$ represent a fluorescent molecule from which radiation of equal intensity eseapes in all directions. Of all the rays leaving $X$ it is clear that only those within the solid cones $\mathrm{EXF}$ and $\mathrm{GXH}$ can escape into the air. The rays within the cones FXH and EXG are imprisoned by total internal reflexion, the ray XJ for example being reflected back and forth between the parallel surfaces of the film. Silvering the lower surface will cause the rays in the cone GXH to escape through the upper surface, but will not affect the paths of the other rays. If now we bring a white matt surface into optical contact with the lower surface of the film, it will liberate all of the rays which were totally reflected. Take the ray XJ for example: it is diffusely reflected at $K$, and that portion of the energy within a cone of the same aperture as that of the cone EXF escapes through the upper surface. The rest is totally reflected from the upper surface, and returned to the white surface, when the process is repeated, all of the energy, except that lost by absorption, eventually passing out through the upper surface. The action of the matt surface liberates the energy imprisoned by internal reflexion. If there were no absorption the edges would appear of dazzling brilliancy, as all of the imprisoned energy would be thrown out here. The greater brilliancy of the edges of a fluorescent film was utilized by Becquerel in observing the faint luminosity of certain compounds of uranium, and by Greinacher in the case of the fluorescence of mica flms caused by radiotellurium (Phys. Zeit. 1906).

We may test the above theory of the action of a matt surface in increasing the brilliancy of the fluorescence by making photometric measurements. The ratio of the energy within the two cones of rays which can escape without the aid of the matt surface, to the energy imprisoned by reflexion, 
is represented by

$$
\frac{1-\cos \theta}{\cos \theta}
$$

which is the ratio of the area on a sphere described around the luminous molecule, cut out by the cone of rays EXF, to the area of the hemisphere minus this portion. For celluloid the critical angle is about $40^{\circ}, i . e_{.}, \theta=40^{\circ}$ and

$$
\frac{1-\cos \theta}{\cos \theta}=0.31
$$

in other words, the imprisoned energy is about three times greater than the energy which escapes. Its liberation by the matt surface should therefore give us about fourfold increase in luminosity. This point was tested with the photometer. As a standard source a rhodamine screen (matt) illuminated by the light of the mercury arc was used, its light being passed through two Nicol prisms by which its intensity could be varied by a known amount. It was compared with a rhodamine film in part covering white porcelain, and in part highly polished silver. The photometer used was a very simple but very satisfactory one made by Dr. Pfund, a semicircle of silvered glass taking the place of a Lummer-Brodhun prism. Equality of luminosity of the two fields of the instrument, with disappearance of the dividing line, was secured in the two cases with the nicols rotated $11^{\circ}$ and $22^{\circ}$ from the position of extinction. Which means that the rhodamine film on porcelain is 3.8 times brighter than the one on silver, which is in good agreement with the calculated value (4). In making the observations a red glass was placed in front of the photometer to exclude everything except the fluorescent light. (White porcelain alone, illuminated by the mercury arc, appeared practically black when viewed through the red glass.) On completing the work it occurred to me that the case was similar to the one to which I drew attention several years ago, namely that a fused bead of microcosmic salt, raised to the highest temperature possible with a blast-lamp, emits little or no light when removed from the flame, though the platinum wire supporting it glows vividly. This is of course a fine case of the failure of a transparent body to radiate, which is what Kirchhoff's law demands. But as the bead cools down it solidifies and numerous cleavage planes appear. At the moment of crystallization it glows with a dull red light, which persists for several seconds. I found difficulty in explaining this (see Phys. Optics, 2nd edition, page 598), but a suggestion 
made by Prof. C. E. Mendenhall certainly gives us a satisfactory hypothesis, though there still remain some points which are not quite clear to me. Professor Mendenball considered that the action of the cleavage planes was merely that they interfered with the imprisonment of radiation by total reflexion. All rays originating within the ball which strike the surface at an angle greater than the critical angle are reflected around the inner surface over and over again, so that they eventually may be considered as having traversed a layer of the medium of infinite thickness. If the medium has any absorption at all, and no media are perfectly transparent, this radiation will be of the same type as that of a black body at the same temperature. Scratches on the surface or internal cleavage planes liberate this energy by interfering with the total reflexion.

Professor Mendenball has suggested that I include some of his observations in the present paper.

He writes me that he made a scratch on the surface of a ball of fused quartz, which was then heated in a Bunsen burner. The black-body temperature of the scratch, as observed with an optical pyrometer, was $850^{\circ} \mathrm{C}$., while that of the rest of the ball was barely $600^{\circ} \mathrm{C}$.

From similar considerations he concludes that a uniformly heated, nearly transparent sphere should appear more luminous towards the limb. (A solid incandescent sphere appears as a uniformly illuminated disk, as a result of the cosine law.) I have verified this with a hollow glass bulb filled with a very dilute solution of rhodamine, illuminated by the mercury arc and viewed through red glass. If a spot on the surface of the bulb is finely ground with flour emery, this spot appears extremely luminous when near the limb, as a result of the liberation of the fluorescent rays which are undergoing total internal reflexion. The bulb appears slightly more luminous at the limb, even when the surface is not roughened. I have observed the same thing in films of celluloid stained with rhodamine, which appear much more luminous when viewed obliquely, at nearly the angle of grazing emergence, than when observed by rays which leave. the surface nearly parallel to the normal.

In regard to the microcosmic bead, I am now of the opinion that most of the light comes from the hot wire. It is difficult to devise a means of supporting a fused bead on a nonluminous support; but I have watched their fall from the fifth floor of the laboratory at night, and have found no evidence of any brightening during the drop of five stories. 